tured as a parallel distributed processing system; (c) invariances: the evaluations of $l_{1}$ and $l_{2}$, invariant under the object's location in the camera's viewing field and its direction of movement, are inherent in the processing structure; (d) approximation vs. exactness: the "relation" property of dimensioning guarantees robust object classifications; (e) generalization: objects that are different from compact rectangles can be selected, too; (f) adaptive properties: for appropriate parameter settings the algorithms allow the net to adapt parameters quickly depending on the global stimulus features provided to the system. The net will be improved, e.g., with respect to the evaluation of spatiotemporal stimulus properties and velocity invariance. Furthermore, the classification of an object will be performed by means of a Kohonen net.

For a simple class of objects, this MODNet was successfully tested in an experimental platform [6] after the problems concerning the interfaces "sensor-
MODNet-actor" were solved (Fig. 4). An object passes an infrared gate that releases the camera whose output processed by the net tells a robot, for example, to grasp only small elongated objects oriented in the belt's direction of movement, and to sort these according to their different lengths. The net's directional invariance allows variable camera positions. The platform will be expanded, so that the net also provides information for the grasping patterns of the robot depending on the dimensions of the object and its position on the belt.

This study is supported by the Bundesministerium für Forschung und Technologie (BMFT), Verbundprojekt "SEKON": Sensorisch-motorische Koordination von Roboterbewegungen mit Neuronalen Netzen, Teilprojekt Nr. 4135839-01 IN 104 C/4 (Ewert), Nr. 4135839-01 IN 104 D/7 (Pfeiffer). Deutsche Forschungsanstalt für Luft- und Raumfahrt (DLR), Projektträger des BMFT für Informationstechnik, Abt. Informati- onsverarbeitung. The authors are responsible for the contents of the publication.

Received March 26, 1993

1. Arbib, M. A.: The Metaphorical Brain 2: Neural Networks and Beyond. New York: Wiley 1989

2. Ewert, J.-P., Capranica, R. R., Ingle, D. J.: Advances in Vertebrate Neuroethology. New York: Plenum 1983

3. Eckmiller, R., Hartmann, G., Hauske, G. (eds.): Parallel Processing in Neural Systems and Computers. Amsterdam: North-Holland/Elsevier 1990

4. Mallot, H. A., v. Seelen, W., in: Visuomotor Coordination: Amphibians, Comparisons, Models and Robots (J.P. Ewert, M. A. Arbib, eds.). New York: Plenum 1989

5. Ewert, J.-P.: Behav. Brain. Sci. 10, 337 (1987)

6. Cruse, H., Ewert, J.-P., Ritter, H., Pfeiffer, F, in: Status Seminar Neuroinformatik (BMFT, Hrsg.) (in press)

\title{
A New Function for an Old Structure: the "Timbal Muscle" in Cicada Females
}

\section{R. M. Hennig, T. Weber, F. Huber, and H.-U. Kleindienst \\ MPI für Verhaltensphysiologie, D-82319 Seewiesen}

\section{T. E. Moore}

University of Michigan, Ann Arbor, Michigan, USA

\section{A. V. Popov}

Sechenov Institute, Russian Academy of Sciences, St. Petersburg (Leningrad), Russia

A prerequisite for an evolutionary change of function is the capacity of a morphological structure to perform two (motor) tasks [1]. To what extent then is the previous neuronal machinery associated with the use of the structure retained, reduced, or modified for the new task [2, 3]? Cicadas may provide an excellent example where such a question can be addressed: in cicadas it is proposed that originally both sexes produced sound signals (as is still the case in many of the cercopidae; [4]). The recent cicada females have lost the ability to produce sound, but they still possess a timbal montoneuron $[5,6]$. This ,timbal motoneuron" innervates a muscle which, together with two other muscles, serves a different function, i.e., the tensor tympani muscle which acts on the tympanal membrane which most likely leads to a modulation of the auditory threshold [6, 7]. In males, too, there is evidence that the timbal muscle helps to perform the task of tympanal relaxation by its tonic component during singing [8]. Here, we compare the motor programs in males and females associated with the common motoneuronal pathway in order to describe possible changes in the motor pattern.

The activity of the auditory nerves, which also carry the axons of the timbal motoneurons in males and females of Okanagana rimosa [5], was recorded using silver hook electrodes $(\varnothing 50 \mu \mathrm{m})$. Singing activity in males was induced by touching the animals at their head, antennae, mouthparts, and various areas of the abdomen or by stimulating the brain electrically [8]. The sounds elicited were sometimes very irregular in time as is not unusual for the disturbance squawks of cicadas. The following analysis is based on the regular sections of the disturbance squawks which was the most com mon pattern elicited in males. Females were also mechanically and electrically stimulated at the same areas as the males and the activity of the auditory nerves recorded. Furthermore, auditory thresholds were determined in each female tested for a motor pattern expression using brief $(1.3 \mathrm{~ms}$ in duration) loud- 
speaker clicks. The auditory nerve response was digitized and then averaged 30 times.

In males the motor pattern of the disturbance squawk, elicited by touching or by electrical brain stimulation, consisted of very regular spike sequences in each nerve recording (Fig. $1 \mathrm{~A}$ ). Each timbal buckling resulted in the production of two to four sound pulses. The combined activity of the timbal motoneurons driving both timbal muscles produced groups of four to eight sound pulses followed by a short pause (Fig. $1 \mathrm{~A}$ ). In females, a

A male
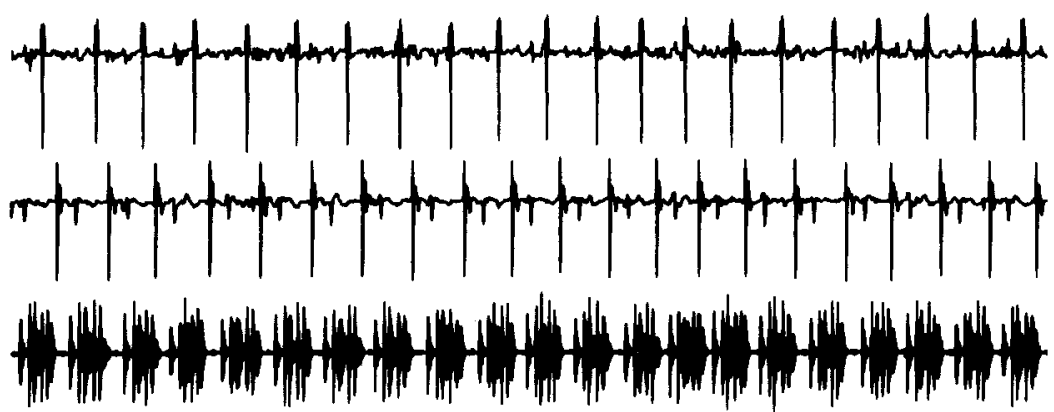

B
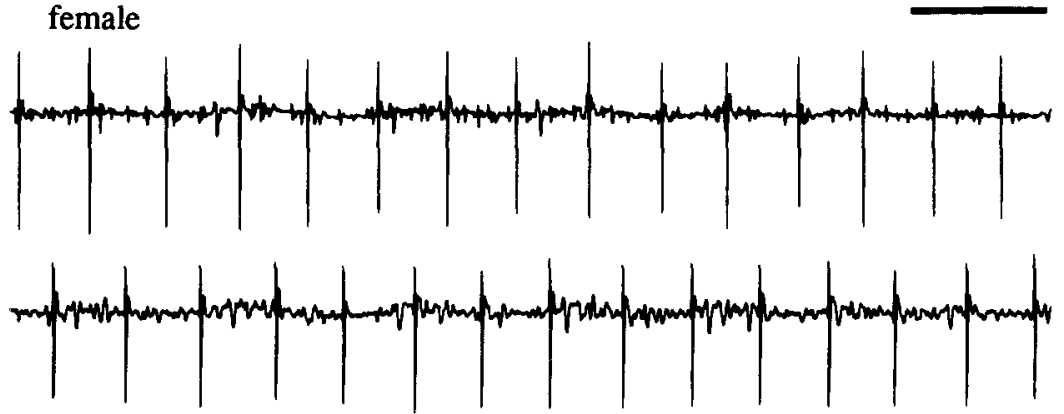

C

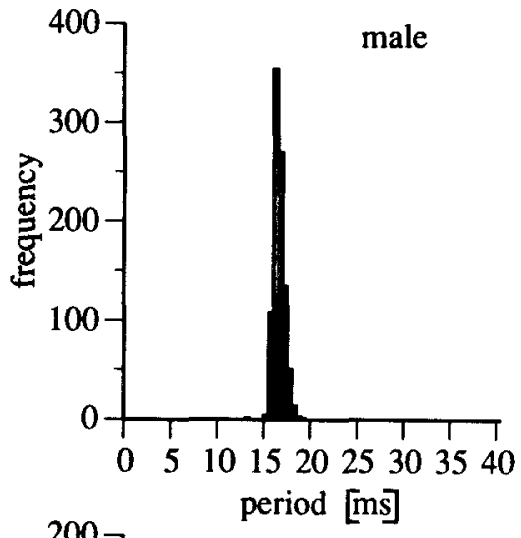

E

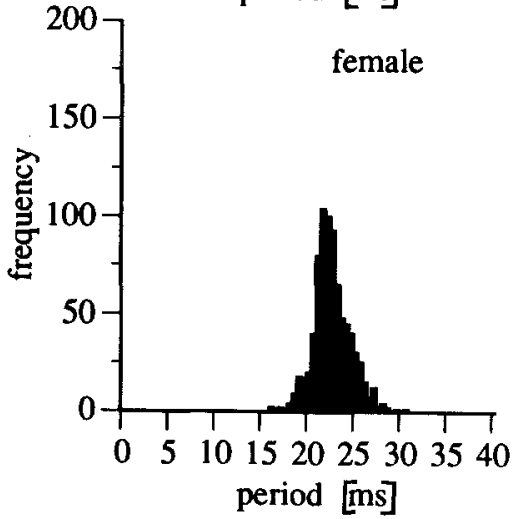

D

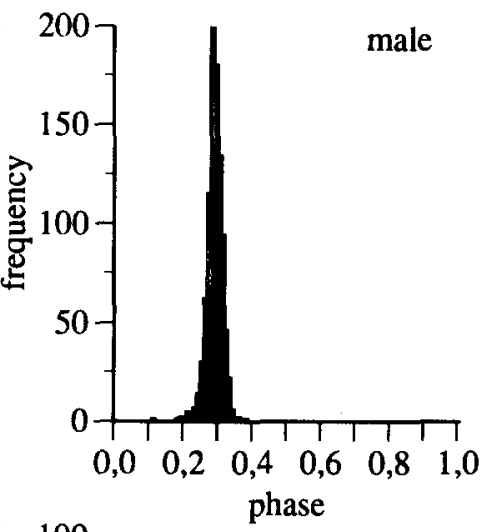

$\mathrm{F}$

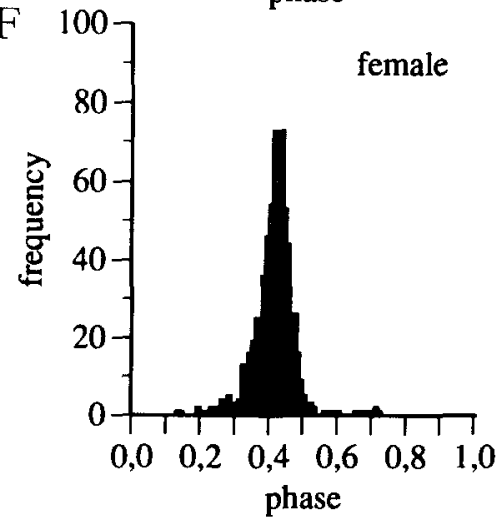

regular pattern of activity of the "timbal motoneuron" was elicited either by touching the females at the same body parts as the males or by electrical brain stimulation (Fig. 1 B). There was always an alternating activity pattern as in the males. The periods and the phase relationships of the motoneuronal activity recorded in the auditory nerve in four males and four females are exemplified in Fig. $1 \mathrm{C}$ to $\mathrm{F}$ for one individual of each sex. Periods in males measured $16.0 \mathrm{~ms}$ (standard deviation $\pm 1.6 \mathrm{~ms}$; Fig. $1 \mathrm{C}$ ) and phase measured 0.29 (standard deviation \pm 0.017 ; Fig. 1 D). In females the periods were longer than in the males $(27.4 \mathrm{~ms})$ and showed a higher standard deviation ( $\pm 6.4 \mathrm{~ms}$; Fig. 1 E). The phase relationship in females was different from the males (0.46) and showed a higher variability (standard deviation \pm 0.041 ; Fig. 1 F). Thus, females can express an activity pattern in the "timbal motoneuron" which is very similar - albeit not the same - as that of the males. The pattern can be elicited by the same manipulations as in males which shows that the sensory pathways involved in eliciting the motor pattern in males also appear to be present in females.

The muscle innervated by the "timbal motoneuron" in cicada females is the tensor tympani which - along with other muscles - probably helps to relax the tympanum which might allow the cicada to adjust its auditory threshold to surrounding noise levels $[6,8]$. In order to test whether the activity of the "timbal motoneuron" has such a motor effect on the tympanum, we stimulated the fe-

Fig. 1. Recordings of both auditory nerves which carry the axon of the timbal motoneuron in males and a likely homologue in females [6]. A) Males show a regular motor pattern when touched or when electrically stimulated at the brain (upper and middle trace: auditory nerve recordings, bottom trace: sound). B) Females show a similar pattern of activity as the males when touched at the same areas or stimulated electrically (upper and lower trace: auditory nerve recordings). Time scale for (A, B) $50 \mathrm{~ms}$. C-F) Histograms of period distributions and phase relationship of the two timbal motoneurons in males and females measured from recordings as in (A) and (B). Examples shown are from one male $(C, D)$ and one female $(E, F)$. Means and standard deviations of all animals tested are given in the text 
males acoustically (loudspeaker clicks from 30 to $80 \mathrm{~dB}$ ) while the motor pattern was recorded from the auditory nerve. A comparison of the magnitude of the auditory nerve response with and without "timbal motoneuron" activity showed that there was a small reduction in the response amplitude when the motoneuronal pattern was present (Fig. 2; data from four females).

In conclusion, the $O$. rimosa females possess not only the timbal motoneuron from song production abilities of their ancestors but are also able to express a song-like - albeit not $O$. rimosa-specific - motor pattern. The sensory pathway initiating the song pattern in males is also present in the females, although details are not known for either sex. The rather pronounced change in structure is accompanied by rather subtle changes in the neuronal system as is also described for grasshoppers [2] and phasmids [9]. The ancestral timbal motoneuron and muscle in cicada females have apparently undergone a change in function from sound production to auditory threshold modulation. The persisting

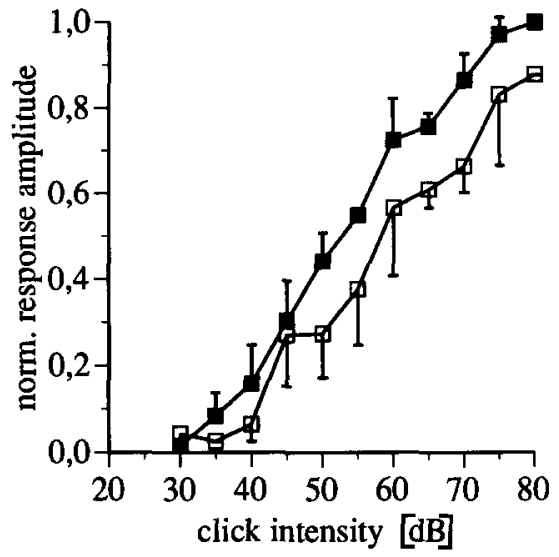

Fig. 2. Comparison of the normalized auditory nerve response to loudspeaker clicks from four females each with ( $\square$ ) and without (a) timbal motoneuron activity. Symbols represent mean values, horizontal bars show standard deviations

"old" motor program associated with sound production in females is a challenge for further research, because it is still not known how the "old" motor pattern is incorporated in the "new" motor control task.
We thank K. Schildberger and G. Horseman for their valuable comments on the manuscript.

Received March 1, 1993

1. Mayr, E.: Animal Species and Evolution. Cambridge, MA: Harvard Univ. Press 1963

2. Arbas, E. A.: J. Comp. Neurol. 216, 369 (1983)

3. Dumont, J. P. C., Robertson, R. M.: Science 233, 849 (1986); Arbas, E. A., Meinertzhagen, I. A., Shaw, S. R.: Annu. Rev. Neurosci. 14, 9 (1991)

4. Ossiannilsson, F: Opusc. Entomol. Suppl. 10, 1 (1949)

5. Wohlers, D. W., Williams, J. L. D., Huber, F., Moore, T. E.: Cell Tissue Res. 203, 35 (1979)

6. Wohlers, D. W., Bacon, J.: ibid. 209, $371(1980)$

7. Pringle, J. W. S.: J. Exp. Biol. 31, 525 (1954)

8. Hennig, R. M., Weber, T., Huber, F., Kleindienst, H. U., Moore, T. E., Popov, A. V.: in preparation

9. Kutsch, W., Kittmann, R.: J. Comp. Physiol. A 168, 483 (1991)

Naturwissenschaften 80, 326-328 (1993) $\mathfrak{C}$ Springer-Verlag 1993

\title{
Valva-Opening Response Induced by the Light Stimulation of the Genital Photoreceptors of Male Butterflies
}

\author{
K. Arikawa \\ Department of Biology, Yokohama City University, 22-2 Seto, Kanazawa-ku, \\ Yokohama 236, Japan
}

Butterflies can detect light by the genitalia as well as by the compound eyes. The photosensitivity of the genitalia is mediated by two pairs of extraocular photoreceptors. Unlike earlier examples of arthropod extraocular photoreceptors that are localized within the central nervous system [1-3], the butterfly genital photoreceptor is the first conclusive example of a peripheral photosensory neuron in arthropods [4]. While the response characteristics [5] and the ultrastructure [6] of the genital photoreceptors have been studied so far, the functional aspects of the unique system are not yet known.
In response to light stimulation of the genitalia, there are some movements of the abdominal segments. For example, when the butterfly curls the abdomen, the light stimulation of the genitalia causes the extension of the abdomen. We previously identified a neuronal circuit in the last abdominal and the penultimate ganglia where photoreceptor activity inhibits some abdominal motoneurons that innervate longitudinal muscles [7]. The circuit apparently constitutes a part of the mechanism of the abdominal extension response. Analysis of the neuronal mechanism underlying such a lightinduced behavior may serve to elucidate the functional significance of the photoreceptive system.

In response to light stimulation of the genitalia, butterflies not only release the curled abdomen but also exhibit various other local movements of the genitalia. This report describes one such movement in male, the valva-opening response (VOR), and demonstrates that the VOR is directly induced by the activity of the genital photoreceptors. Since the valva is a specialized structure for copulation, our results suggest that the genital photoreceptive system has some role in controlling the copulation behavior.

Figure 1 shows the VOR in an intact male Papilio protenor. The photographs were taken from the ventral side without (a) and with (b) illumination from the posterior end of the animal. The butterfly opens the valvae widely in the presence of light. The movement of valvae observed here always occurred symmetrically, i.e., bilateral valvae open or close simultaneously.

The photoreceptive sites exist on the scaphium (photoreceptive site 1, P1) and on the diaphragm (P2), both of which are 